\title{
Use of experimentation in the accurate numerical simulation of thermal processes
}

\author{
Y. Jaluria \\ Mechanical and Aerospace Engineering Department, Rutgers University, \\ Piscataway, NJ, USA
}

\begin{abstract}
Experimental results play a crucial role in the validation of mathematical and numerical models for a variety of thermal transport problems. Material properties that are crucial to any accurate simulation are obtained experimentally. In addition, there are many important processes where the boundary conditions are not well defined. However, experimental work can be used to provide the appropriate boundary conditions, or the numerical solution of a coupled inverse problem can be used to define these for an accurate simulation. Finally, there are many problems in which numerical simulation is particularly suitable over given parametric ranges, while experimentation is more appropriate over other regions. In such cases, a concurrent numerical and experimental approach may be used to solve the problem more accurately and efficiently. The basic considerations that arise in these approaches are outlined. A few circumstances, where a coupling of the experimentation with the simulation is appropriate, or where experimentation is used to define the boundary conditions are presented.
\end{abstract}

Keywords: numerical simulation, thermal processes, systems, experimentation.

\section{Introduction}

Numerical modeling and simulation of basic and applied problems is extensively used in thermal systems to understand the underlying phenomena and design and optimize the system. Numerical simulation is of crucial importance because of the limited applicability of analytical methods, particularly for practical applications which are generally quite complicated, and the expense and time involved in experimental methods, see Jaluria [1]. The model is often validated by the use of analytical and experimental results, if available. Experimental 
results are also valuable for physical insight and basic understanding. However, experiments are also needed in many problems to initiate the numerical simulation, to obtain greater accuracy and efficiency, or to provide necessary inputs for accurate results. This paper focuses on circumstances where the simulation is strongly dependent on or coupled with experimental data for accurate results.

Thus, this review paper focuses on the following aspects:

- Material Properties and Characteristics

- Verification and Validation

- Experimentally Determined Boundary Conditions

- Solution of Inverse Problems with Experimental Inputs

- Concurrent Numerical Simulation and Experimentation

Experimental results are needed to provide material properties needed for simulation, as well as data for validation of the models. Experimental results are also often needed to define and determine the boundary conditions. In some cases, experiments can be obtained only over a limited domain and an inverse problem has to be solved to obtain the desired results. Finally, there are regions where experimentation is more accurate and efficient than numerical simulation, whereas the latter may be more appropriate in other regions. Then, a concurrent use of experimentation and numerical simulation can solve the problem most efficiently and accurately.

There are many thermal transport problems for which numerical simulation has been used to provide inputs for design and for understanding the basic processes involved. Examples of these include heat rejection from the condensers of a power plant to a water body such as a lake, electronic systems consisting of isolated heat sources in an enclosure with forced flow driven by a fan, materials processing applications such as chemical vapor deposition (CVD) in which chemical reactions result in the deposition of a thin film on a hot susceptor and a room with a fire resulting in a stratified hot upper layer in the room due to the fire plume and flow exchange through an opening. Because of complexities such as turbulent flow, combined heat and mass transfer, chemical reactions, complicated geometry, variable material properties, and buoyancy effects, numerical simulation is needed to model the transport in such thermal processes.

\section{Material properties and characteristics}

Accurate material properties are crucial to the validity and usefulness of any numerical simulation. However, accurate property data are often not available and the simulation has to employ the information available. Frequently, the data are available at conditions that may be different from those for the actual process. For instance, the fabrication of optical fibers employs the heating of a cylindrical silica glass preform in a furnace to a temperature above its softening point $T_{\text {melt }}$, which is around $1900 \mathrm{~K}$, and drawing it into a fiber through a neckdown region, as reviewed by Paek [2]. The neck-down depends strongly upon 
the operating conditions and the physical properties of silica glass. However, these are strong functions of the temperature $T$. The variation in the viscosity is the most critical one for the flow, since it varies exponentially with temperature. A curve fit of available data for kinematic viscosity $v$ is obtained for silica, in S.I. units, as

$$
v=4545.45 \exp \left[32\left(\frac{T_{\text {melt }}}{T}-1\right)\right]
$$

The radiative source term $S_{r}$ in the energy equation is non-zero for the glass because it emits and absorbs thermal energy. The variation of the absorption coefficient with wavelength $\lambda$ has been measured for certain glasses, as shown in fig. 1. However, the data are often obtained at room temperature, whereas the process is at much higher temperatures. Still, such data are valuable and are used in the simulation, with the continuous variation being approximated as bands with constant absorption over each band to simplify the simulation. A two- or three-band absorption coefficient distribution can be used, as shown in fig. 1. Then, the zonal method or the discrete ordinates method can be used to model the radiative transport, as discussed by Yin and Jaluria [3]. Other more accurate methods are also available, though the major constraint is the availability of accurate property data.

Similarly, the transport phenomena in polymer processing involve large material property changes, particularly with temperature and species concentration. Most polymers are also non-Newtonian and the viscosity varies with the shear rate and thus with the flow, making the problem even more complicated. The changes in the chemical structure for materials like food can also be included if experimental data are available.
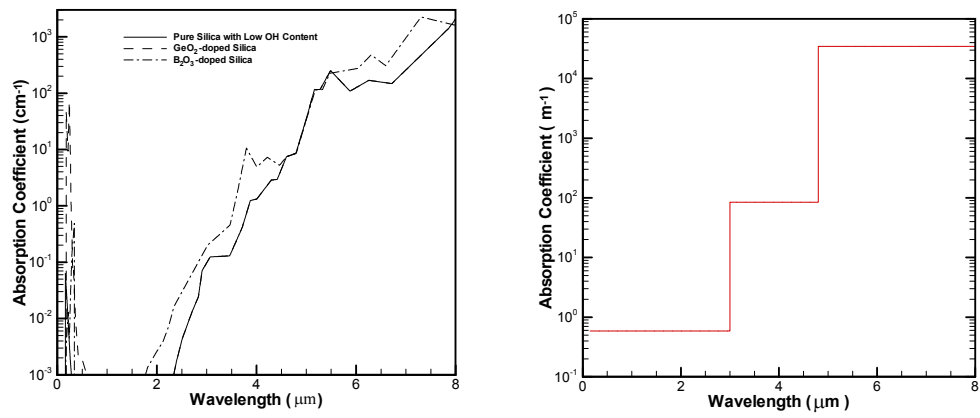

Figure 1: $\quad$ Experimentally measured absorption coefficient of silica glass as a function of the wavelength and the approximation in terms of a band model.

\section{Verification and validation}

Verification and validation are particularly important considerations in any numerical simulation, where verification ensures that the numerical code 
performs satisfactorily and validation ensures that the model is an accurate representation of the physical problem, see Roache [4]. Grid refinement is an important aspect in verification. The results are considered in terms of the physical behavior of the system to confirm that the observed trends are physically reasonable. A comparison with available analytical and numerical results, particularly benchmark solutions, can be used for validating the model. Experimental data may be available on similar systems and may also be used for validation. However, it may be necessary in some cases to develop an experimental arrangement for providing inputs for validation. Though this is not a trivial exercise, it could be crucial to establishing the validity of the models, particularly for complex systems if no other information is available.

As an example, consider polymer processing in twin-screw extruders. Experiments have been carried to validate the model, particularly the transport in the intermeshing region between the two rotating screws. The basic phenomena are not very well understood and an experimental system consisting of two rotating cylinders was developed. The mixing process in the intermeshing region was investigated, as discussed by Sastrohartono et al. [5]. Experimentally and numerically obtained streamlines in the region between two rotating cylinders, approximating the twin-screw extruder, are shown in fig. 2, indicating good agreement. Some of the fluid flowing adjacent to the left cylinder continues to flow along its surface, while the remaining flows over to the other cylinder. A flow division ratio $x_{f}$, defined as the fraction of the mass flow that crosses over from one channel to the other, is taken as a measure of mixing and is determined by using the dividing streamline that separates the two fluid streams. A comparison between experimental and numerical results is shown, indicating good agreement at the typically small Reynolds numbers encountered in extruders. For more detailed comparisons, velocity and temperature distributions
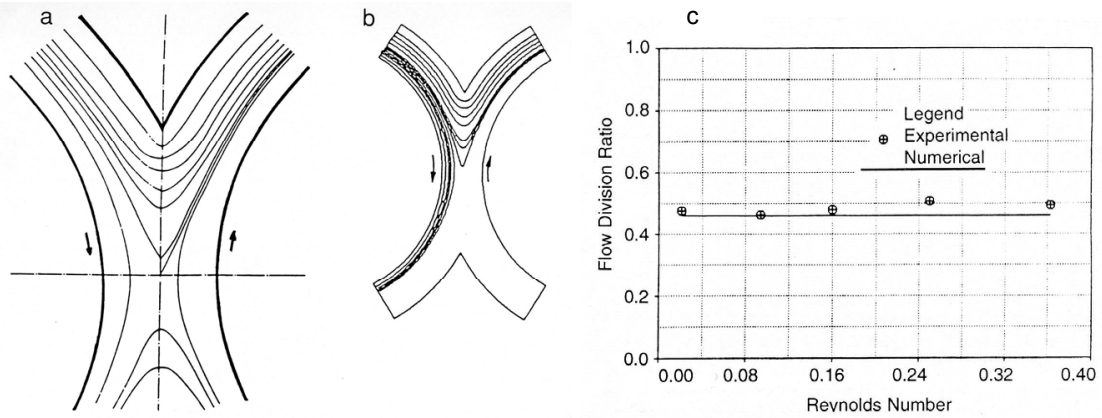

Figure 2: $\quad$ Streamlines in the region between two rotating cylinders for CMC solution at $16 \mathrm{rpm}$. (a) Experimental results; (b) Numerical predictions for flow entering the region over one cylinder; (c) Comparison of flow division ratio $x_{f}$ obtained from experimental and numerical results. 
have been measured using more elaborate experimental systems and compared with the numerical results [6].

\section{Experimentally obtained boundary conditions}

In many practical problems, the boundary conditions are complicated and are obtained experimentally to simplify the problem. Simulations with boundary conditions given as convective with a specified heat transfer coefficient $h$, which is obtained from empirical correlations based on separate experiments, are quite common. If the heat transfer coefficient is not specified, the solution could involve complex domains and conjugate problems and could thus be substantially more complicated. Similarly, the neck-down profile in optical fiber drawing is an unknown and is the result of the various forces acting on the fiber. These include viscous, gravitational, surface tension and shear forces at the surface. This is a fairly involved problem and requires substantial computational resources, time and effort. Therefore, several investigators assumed the neckdown profile on the basis of experimental data to substantially simplify the analysis.

The coating of optical fibers involves the fiber moving at high speed through a reservoir of the coating fluid, which is often an acrylate, and then curing the polymer coating obtained. This generates an inlet meniscus, whose characteristics, particularly its stability, have been studied in detail because air entrapment by the moving fiber and the resulting deterioration of the coating are largely due to the breakdown of the meniscus. The problem is very complicated because it involves a dynamic contact angle, with the fiber moving at speeds as high as $20 \mathrm{~m} / \mathrm{s}$ through the air and then plunging into the liquid, with air being replaced by the liquid in the boundary layer, see Quere [7]. It is a difficult problem to model analytically, but the meniscus is needed to solve for the flow in the coating applicator.

In order to considerably simplify the problem, the shape and dimensions of the meniscus may be prescribed on the basis of experimental data, such as the experimental results obtained by Ravinutala and Polymeropoulos [8] and shown in fig. 3 , in terms of images of the meniscus. It was found that the effect of the fluid pressure is to flatten the meniscus and to increase the slope of the liquid-air interface near the fiber compared to those for an unpressurized meniscus. Several other such results were obtained and the meniscus determined for a range of operating conditions, using glycerin as the fluid, which was also employed in the numerical simulations. Assuming axisymmetric flow, the governing equations are given in terms of velocity $u, v$ in radial $r$ and axial $z$ directions, respectively, pressure $p$, temperature $T$, and time $t$ as the following equations, with $\Phi$

representing viscous dissipation, $S_{r}$ the source term, $K$ the thermal conductivity, $v$ the kinematic viscosity, $\rho$ the density and $C_{p}$ the specific heat at constant pressure [9]:

$$
\frac{\partial v}{\partial z}+\frac{1}{r} \frac{\partial(r u)}{\partial r}=0
$$




$$
\begin{gathered}
\frac{\partial v}{\partial t}+u \frac{\partial v}{\partial r}+v \frac{\partial v}{\partial z}=-\frac{1}{\rho} \frac{\partial p}{\partial z}+\frac{1}{r} \frac{\partial}{\partial r}\left[r v\left(\frac{\partial v}{\partial r}+\frac{\partial u}{\partial z}\right)\right]+2 \frac{\partial}{\partial z}\left(v \frac{\partial v}{\partial z}\right) \\
\frac{\partial u}{\partial t}+u \frac{\partial u}{\partial r}+v \frac{\partial u}{\partial z}=-\frac{1}{\rho} \frac{\partial p}{\partial r}+\frac{2}{r} \frac{\partial}{\partial r}\left(r v \frac{\partial u}{\partial r}\right)+\frac{\partial}{\partial z}\left[v\left(\frac{\partial v}{\partial r}+\frac{\partial u}{\partial z}\right)\right]-\frac{2 v u}{r^{2}} \\
\rho C_{p}\left(\frac{\partial T}{\partial t}+u \frac{\partial T}{\partial r}+v \frac{\partial T}{\partial z}\right)=\frac{1}{r} \frac{\partial}{\partial r}\left(r K \frac{\partial T}{\partial r}\right)+\frac{\partial}{\partial z}\left(K \frac{\partial T}{\partial z}\right)+\Phi+S_{r}
\end{gathered}
$$

Extensive numerical results were obtained by Yoo and Jaluria [9], using finite volume methods for the primitive variables. Figure 3 shows the computed velocity field in the applicator, with a prescribed upper meniscus. Even though the flow near the meniscus changes substantially with a change in the meniscus, the flow far away remained largely unaffected. The results were also compared with experimental measurements and a good agreement between the two was obtained. Thus, a prescribed meniscus considerably simplifies the simulation.

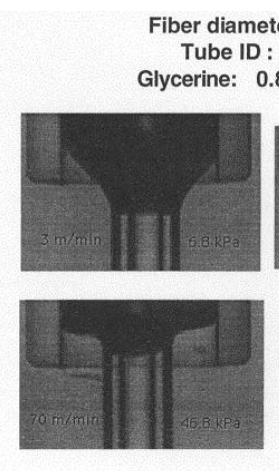

ber diameter : $254 \mu \mathrm{m}$ Tube ID : $460 \mu \mathrm{m}$ Glycerine: $0.88 \mathrm{~N} \mathrm{sec} / \mathrm{m}^{2}$

Figure 3: Experimental images of the inlet meniscus due to an optical fiber entering a reservoir of Glycerin at different pressures and typical numerical results on the flow in the coating applicator with a prescribed meniscus.

\section{Solution of inverse problems with experimental inputs}

In several practical thermal systems, limited access to the flow domain is available, constraining the experimental data that can be obtained through techniques such as laser and infrared diagnostics. An important example is the 
optical fiber drawing furnace, which typically has an infrared sensor to monitor the temperature of the heating element at only one location for control and access to the furnace interior is not easily possible, see Paek [2] and Issa et al. [10]. The temperature distribution at the wall is the result of the overall heat transfer processes in the furnace and cannot be taken as a known input to the model. However, since the temperature affects the fluid properties, as well as the transport mechanisms in the flow, the wall temperature is critical to the calculation of the glass flow and thus to the characteristics of the fiber. In such cases, the limited data that can be obtained by experimentation is used in conjunction with numerical modeling to solve an inverse problem to determine the wall temperature distribution.

Issa et al. [10] used temperature measurements at the centerline of a graphite rod placed at the axis of the furnace to solve an inverse problem that yielded the wall temperature distribution. Each rod was instrumented with thermocouples inserted through an axial hole along the centerline. The temperature measurements were used along with a numerical model for the flow and heat transfer in the furnace in order to obtain the furnace wall temperature profile. This is an inverse problem since the centerline temperature in the rod is known whereas the furnace thermal conditions are not known. An optimization technique was used to ensure that the result of the inverse calculation was essentially unique. The results obtained using the graphite rods indicated that the furnace temperature is not significantly affected by rod size. Figure 4 shows the computed and measured temperature distributions along the graphite heating element. The dashed lines represent the water cooled portion of the furnace cavity. Excellent agreement was observed between the predicted and measured rod temperatures. The computed maximum element temperatures were in good agreement with the furnace sensor temperature at the hot zone centerline, lending support to the model for the flow and thermal transport in the furnace. Similar
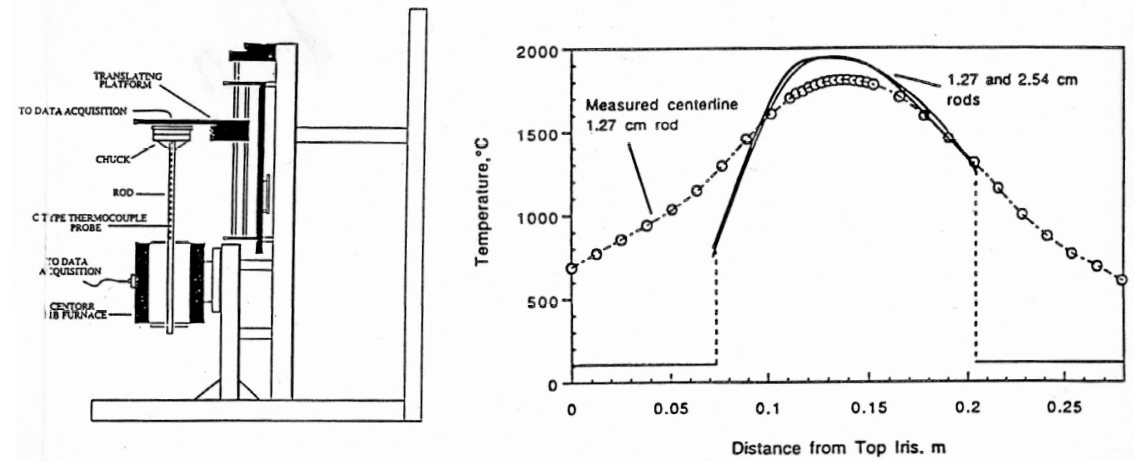

Figure 4: Experimental arrangement for measuring temperatures at the centerline of a graphite rod immersed in a fiber drawing furnace and the numerical results from the solution of the inverse problem to obtain the heating element temperature distribution. 
results were obtained for the other furnace temperatures and for other materials, including silica glass.

\section{Concurrent simulation and experimentation}

Conventional engineering design and optimization are based on sequential use of computer simulation and experiment, with the experiments generally being used for validation or for providing selective inputs. However, these methods fail to use the advantages of using experiment and simulation concurrently in real-time. Numerical simulation can easily accommodate changes in geometry and material, whereas experiments can more conveniently vary the operating conditions like flow rate and heat input. Also, laminar and unstable flows can generally be simulated conveniently and accurately, whereas transitional and turbulent flows are often more accurately treated experimentally. By using concurrent numerical simulation and experimentation, the entire domain of can be considered for system design and optimization efficiently and accurately.

The simple physical system considered is shown in fig. 5. It consists of single and multiple isolated heat sources, which approximate electronic components, located in a horizontal channel in a two-dimensional configuration, as considered, for instance, by Sathe and Sammakia [11]. The one on the left is designated as the $1^{\text {st }}$ heat source. Their height and width are $h$ and $w$, respectively. Heat sources are separated by distance $d$. The thickness of the bottom plate is $B$.

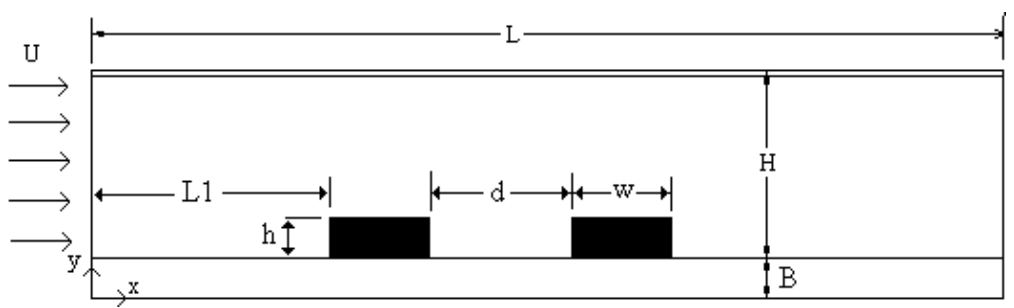

Figure 5: Two heating elements in a channel, simulating an electronic system.

The problem involves removing the energy dissipated by these components by the flow of air or a dielectric liquid. The flow conditions are defined by the Reynolds number $R e$ and the Grashof number $G r$, both being based on the channel height. The fluid is represented by its properties particularly the Prandtl number $P r$. Design variables include channel dimensions, coolant, and location and orientation of the heat sources. Typical design objectives are maximizing the heat removal rate from the components and minimizing the pressure drop. The inputs required for the design and optimization of the system are obtained using numerical simulation for low flow rates and heat inputs and experiment for larger values. The switch from simulation to experiment is determined based on the critical values of $R e$ and $G r$ values for transition to turbulence, as discussed by Icoz and Jaluria [12]. 
The governing non-dimensional differential equations for laminar mixed convection flow, with constant properties can be written in the following form:

$$
\begin{gathered}
\nabla \cdot \vec{V}=0 \\
\frac{\partial \vec{V}}{\partial t}+\overrightarrow{V \cdot \nabla \vec{V}}=-\nabla P+\frac{1}{\operatorname{Re}} \nabla^{2} \vec{V}-\frac{G r}{\operatorname{Re}^{2}} \theta \cdot \vec{g} \\
\frac{\partial \theta}{\partial t}+\vec{V} \cdot \nabla \theta=\frac{1}{\operatorname{Re} \operatorname{Pr}} \nabla^{2} \theta
\end{gathered}
$$

Conduction equation within the bottom plate (substrate):

$$
\frac{\partial \theta}{\partial t}=\frac{1}{\operatorname{Re} \operatorname{Pr}} \nabla^{2} \theta
$$

where $\vec{V}$ is the velocity vector, $\vec{g}$ the gravitational acceleration and $\theta$ the dimensionless temperature. The boundary conditions include fully developed axial and zero vertical velocity at the inlet at ambient temperature, fully developed flow conditions at the exit, no-slip conditions and adiabatic surface assumption at top and bottom walls. These equations are solved using the finite volume method for primitive variables on a non-uniform staggered grid, employing the alternating Direction Implicit (ADI) method for time marching and the pressure calculations carried out using a scheme similar to the SIMPLER algorithm of Patankar [13]. Further details on the numerical scheme and analysis can be obtained from Jaluria and Torrance [14].
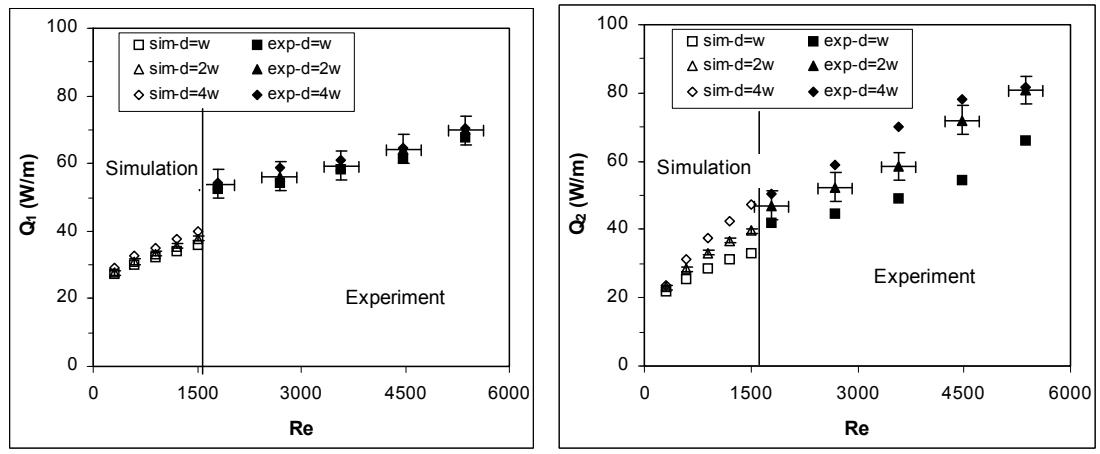

Figure 6: $\quad$ Computed and measured heat transfer rates as functions of $R e$ and $d$ when $h / H=0.25$ from a) first heat source, and b) second heat source.

The temperature and velocity distributions, the heat removal rates and pressure drop are calculated using numerical simulation code for laminar flows, as well as for the beginning of oscillatory flow. Experiments are used for translational and turbulent flows. The first part of the simulation results deals with the determination of the critical flow conditions up to which numerical 
simulation can be used satisfactorily. The critical $R e$ value is determined by observing the transient change in the Nusselt number $N u$ values as a function of time for different $R e$ values. Then, the numerical and experimental results are combined for the design optimization of the air-cooling system. First, the agreement between the results obtained using the two methods is studied. Figure 6 shows the computed and measured results. A jump, in the first heat source heat transfer rate, is observed when the switch from simulation to experiment occurs. This is attributed to the turbulence in the incoming airflow and three-dimensional effects. For the $2^{\text {nd }}$ heat source heat transfer rate, very good agreement is found between the numerical and experimental results, since the wakes created by the first source are very well modeled numerically.

Figure 7 shows the two regions for simulation and experimentation in the physical domain of the problem shown in fig. 6 . The overlapping region between the two is used for validation and for switching from one approach to the other. The resulting optimization of the process in terms of the ratio of the temperature to the pressure drop for varying flow rates is also shown. For the uniform heat flux condition considered here, a minimum in the temperature indicates a maximum in the heat flux. The computed pressure drop results were also found to be in reasonable agreement with experimental data. The experimental values were found to be about $15 \%$ less than the computational results at around $R e=$ 1500. The effect of separation distance on the pressure was found to be small, increasing $\triangle P$ less than $8 \%$. Similarly, several other results and trends were investigated using concurrent numerical simulation and experimentation.

The increase in the heat transfer due to the presence of a passive vortex promoter in the channel flow was also considered [15] and concurrent simulation and experimentation methodology was used to obtain the heat transfer and pressure results, the former being shown in fig. 8. This figure shows the heat transfer rates $Q_{1}$ and $Q_{2}$ for the two sources obtained from numerical simulation at low $R e$ and from experiment at large $R e$. There is fairly good agreement between the two in the overlapping region, though there is uncertainty in the experimental data as indicated here. These results were used to generate the response curves, from which optimal conditions were obtained. The shape of the


Figure 7: Physical domain for optimization with numerical and experimental inputs and the resulting optimization with a composite objective function, which includes heat transfer and pressure drop. 

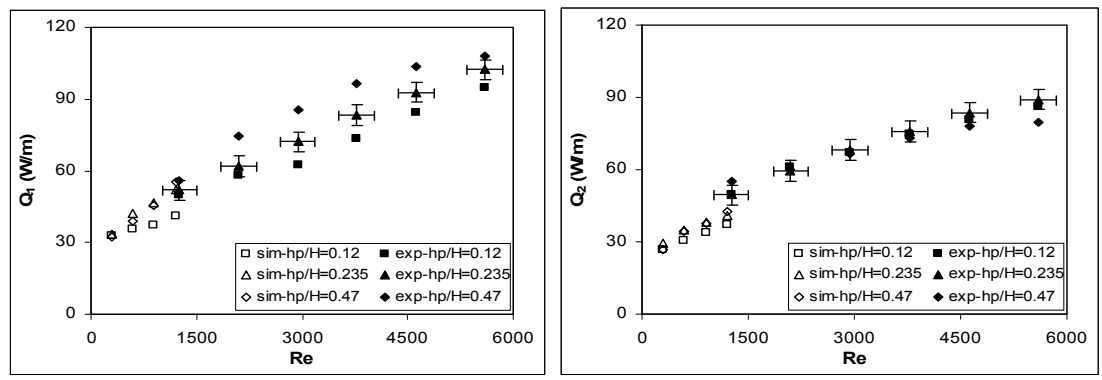

Figure 8: Results for two isolated heat sources in forced flow in a channel with a vortex promoter.

promoter was also taken as a parameter, along with the channel and source geometry. The operating conditions were also varied. Other optimization strategies were also used, but the input data needed for all these were obtained by concurrent simulation and experimentation. For additional results and further details, see Icoz and Jaluria [15], Zhao et al. [16] and Darema [17].

\section{Conclusions}

Experimentation is needed in a wide range of basic and applied heat transfer problems to provide inputs for accurately defining the boundary conditions, simplifying the modelling, and obtaining results over domains where simulation is inaccurate or inefficient. This paper presents circumstances where the numerical simulation is efficiently coupled with experimentation and dependent on experimental data. Examples of such problems are given and the difficulties with specifying the boundary conditions as well as with simulating the entire domain are outlined. Approaches for experimental data driven simulation are discussed and results are presented for several simple and complex problems. It is shown that such approaches are critical to an accurate numerical simulation in many cases of practical interest. The basic methodology is presented and the current status is discussed. Areas where further work is needed are also briefly brought out.

\section{Acknowledgements}

The author acknowledges the support of the National Science Foundation, through several grants, for much of the work reported here. The author also acknowledges the interactions with Professors D. Knight, T. Rossmann and C. Polymeropoulos, and the work done by several students, as given in the references. 


\section{References}

[1] Jaluria, Y., Design and Optimization of Thermal Systems, Second Edition, CRC Press, Boca Raton, FL, 2008.

[2] Paek, U.C., Free drawing and polymer coating of silica glass optical fibers. ASME Journal of Heat transfer, 121, pp. 774-788, 1999.

[3] Yin, Z. \& Jaluria, Y., Zonal method to model radiative transport in an optical fiber drawing furnace. ASME J. Heat Transfer, 119, pp. 597-603, 1997.

[4] Roache, P.J., Verification and Validation in Computational Science and Engineering, Hermosa Publishers, Albuquerque, New Mexico, 1998.

[5] Sastrohartono, T., Esseghir, M., Kwon, T.H. \& and Sernas, V., Numerical and experimental studies of the flow in the nip region of a partially intermeshing co-rotating twin screw extruder, Polymer Engg. Sci., 30, pp. 1382-1398, 1990.

[6] Jaluria, Y, Thermal processing of materials: From basic research to engineering, ASME J. Heat Transfer, 125, pp. 957-979, 2003.

[7] Quere, D., Fluid coating on a fiber, Ann. Rev. Fluid Mech., 31, pp. 347-384, 1999.

[8] Ravinutala, S. \& Polymeropoulos, C.E., Entrance meniscus in a pressurized optical fiber coating applicator, Int. J. Exp. Heat Transfer Fluid Mech., 26, pp. 573-580, 2002.

[9] Yoo, S.Y. \& Jaluria, Y., Fluid flow and heat transfer in an optical fiber coating process, Int. J. Heat Mass Transfer, 50, pp. 1176-1185, 2007.

[10] Issa, J., Yin, Z., Polymeropoulos, C.E. \& Jaluria, Y., Temperature distribution in an optical fiber draw tower furnace, Journal of Materials Processing and Manufacturing Science, 4, pp. 221-232, 1996.

[11] Sathe, S., \& Sammakia, B., A review of recent developments in some practical aspects of air-cooled electronic packages, ASME J. Heat Transfer, 120, pp. 830-839, 1998.

[12] Icoz, T., \& Jaluria, Y., Design of cooling systems for electronic equipment using both experimental and numerical inputs, ASME J. Elec. Packaging, 126, pp. 465-471, 2005.

[13] Patankar, S.V., Numerical Heat Transfer and Fluid Flow, Taylor and Francis, New York, 1980.

[14] Jaluria, Y. \& Torrance, K.E., Computational Heat Transfer, Second Edition, Taylor \& Francis Pub. Co., New York, 2003.

[15] Icoz, T. \& Jaluria, Y., Design optimization of size and geometry of vortex promoter in a two-dimensional channel, ASME J. Heat Transfer, 128, pp. 1081-1092, 2006.

[16] Zhao, H., Icoz, T., Jaluria, Y. \& Knight, D., Application of data driven design optimization methodology to a multi-objective design optimization problem, J. Engg. Design, 18, pp. 343-359, 2007.

[17] Darema, F., Dynamic data driven application systems: A new paradigm for application simulations and measurements, in 4th International Conference on Computational Science, Springer-Verlag, Berlin, pp. 662-669, 2004. 